\title{
Advances in Pattern Recognition Algorithms, Architectures, and Devices
}

\author{
Mohammad S. Alam, FELLOW SPIE \\ University of South Alabama \\ Department of Electrical and Computer \\ Engineering \\ 307 North University Boulevard \\ Mobile, Alabama 36688-0002 \\ E-mail:malam@usouthal.edu \\ Mohammad A. Karim, FELLOW SPIE \\ Old Dominion University \\ Office of the Vice President for \\ Research \\ 2035 Hughes \\ Norfolk, Virginia 23529 \\ E-mail: mkarim@odu.edu
}

Over the last decade, tremendous advances have been made in the general area of pattern recognition techniques, devices, and algorithms. We have had the distinct pleasure of witnessing this remarkable growth as evidenced through their dissemination in the previous Optical Engineering special sections we have jointly editedJanuary 1998, March 1998, May 2000, and January 2002. Twenty-six papers were finally accepted for this latest special section, encompassing the recent trends and advancements made in many different areas of pattern recognition techniques utilizing algorithms, architectures, implementations, and devices. These techniques include matched spatial filter based recognition, hit-miss transforms, invariant pattern recognition, joint transform correlator (JTC) based recognition, morphological processing based recognition, neural network based recognition, wavelet based recognition, fingerprint and face recognition, data fusion based recognition, and target tracking, as well as other techniques. These papers summarize the work of 70 researchers from eight countries.

The first group of papers addresses the issues pertaining to matched filtering. In the first paper, Sims and Mahalanobis discuss the application of a novel quadratic filtering method using missile seeker infrared closing sequences. An important advantage of this technique is how the throughput complexity of the filtering approach, in the detection process, does not vary with scene content. In the second paper, Khoury, Gianino, and Woods investigate the heterocorrelation of different images solely by modifying the filter's intensity transmissivity in the areas of greatest phase mismatch, relative to the phase of the stored template. This approach can also convert a single object recognition filter to a multiple object classification filter. In the third paper, Chen, Zhang, and Karim propose an image compression algorithm to achieve a high compression ratio while simultaneously enhancing correlation-based pattern recognition performance. This is done by emphasizing middle and high frequencies, while discarding low-frequency components through the use of a new distortion measure for compression. Then, Power proposes a new approach, called multiple joint comparative normalized measures (MJCNM), which compares the object and template jointly and then creates a normalized measure for matching. Confidence in the match is shown to be better against certain shape variability by using the MJCNM approach as opposed to using one particular shape measure. In particular, the MJCNM approach uses matched filter, Procrustes, partial-directed Hausdorff, and percent-pixels-same measures. Experimental results demonstrate the usefulness of the multiple comparative measure approach for recognizing object shapes.

Joint transform correlation is the topic of the next group of papers, where Widjaja and Suripon propose a JPEG-compressed reference image based joint transfer correlator (JTC) technique to improve time response of automatic target recognition systems. This technique has been found to be robust to noise and contrast variation for a wide range of compression levels. Thereafter, Alsamman and Alam propose an extremely fast technique for multiwavelet analysis using a phase-encoded JTC. In this technique, mutiwavelet analysis is performed in a single correlation by multiplexing in-phase reference wavelets. Then, Jutamulia, Mu, and Song investigate the application of laser diodes in JTC implementation by con- 
sidering related factors such as temporal coherence, spatial coherence, astigmatism, and elliptical beams.

Robust target recognition using wavelet transform based processing is the topic of the following paper. Jedynski and Chalasinska-Macukow propose an optical pattern recognition technique that combines the performance of wavelet transform and a multilevel composite filtering. This technique yields better distortion invariance by extracting crucial ridge information while eliminating its redundant features.

The next group of papers deals with pattern recognition using adaptive target recognition techniques. In the first paper, Young et al. propose an adaptive target detection algorithm for forward-looking infrared (FLIR) imagery, based on measuring the difference of structural information within the target and the surrounding background. This approach efficiently utilizes eigenspace transform and principal component analysis. Then Du et al. propose a new metric based on the spectral information divergence and spectral angle mapper. This measure has been found to be effective for spectral characterization of multispectral and hyperspectral signatures for material identification. Thereafter, Chang et al. propose a novel data fusion technique for hyperspectral and synthetic aperture radar (SAR) image classification. This technique has been developed for land cover classification, based on the fusion of remote sensing images of the same scene collected from multiple sensors.

The next paper deals with distortion-invariant pattern recognition using a hit-miss transform. In this paper, Doh et al. proposed a new synthetic hit-miss transform (SHMT) algorithm for distortion-invariant recognition of various objects in noisy and cluttered input images. The proposed SHMT algorithm uses synthetic structuring elements, which are synthesized based on a synthetic discriminant function, and has shown the ability to detect various objects with distortions in noisy and cluttered scenes.

Biometric recognition and pattern authentication is the subject of the next group of papers. In the first paper, Casasent and Yuan investigate face recognition by considering both pose and illumination variations using SVRDM support vector machine. In the second paper, Hong, Fei-jun, and Widjaja consider a new method for fingerprint identification by using ANFIS-based matching algorithm, where Gabor transform is used to extract features of fingerprints, while ANFIS is trained to identify the resultant Gabor features. In the third paper of this group, Venkataramani and Kumar show how biometric authentication can provide an added level of security in access control applications. In this paper, the authors evaluate the performance of advanced correlation filters for fingerprint verification using the National Institute of Standards and Technology Special Database 24, obtained from an optical fingerprint sensor, in the presence of various distortions. Thereafter, Birch et al. propose a new system for automating the enforcement process using color image segmentation techniques to find the wind-screen area, and face detection to determine the number of occupants within a vehicle to enforce high-occupancy vehicle lanes. Finally, Ahmed proposes a correlation-based digital watermarking technique for robust image pattern authen- tication. Authentication performance is measured by a correlation test of the extracted signature and the signature computed from the marked image. The quality of the marked image is obtained from the peak signal-to-noise ratio metric.

Neural network based pattern recognition is the topic of the next paper. In this paper, Kypraios et al. propose a hybrid optical neural network (HONN) filter by combining the optical implementation and shift-invariance of correlator-type filters with the nonlinear superposition capabilities of artificial neural network methods. This paper presents the design and implementation of the HONN filter architecture and assesses its object recognition performance in the presence of clutter.

Pattern recognition using holographic techniques is the subject of the next group of papers. In the first paper, Kim and Poon investigate depth detection and image recovery of a target from its complex hologram. The authors report experimental verification of the technique by optical acquisition of the complex hologram of the remote target and subsequently extract the target's depth. The next paper by Shahriar et al. demonstrates a super-parallel holographic correlator which can be operated either as the super-parallel holographic optical correlator or as a superparallel holographic random access memory, with a minor reorientation of some of the elements in real time. This hybrid device eliminates the need for a separate random access memory for holographic correlator-based target recognition and tracking system. Thereafter, Haji-Saeed et al. demonstrate a photorefractive real-time holographic deconvolution technique for adaptive one-way image transmission through aberrating media. This technique can simultaneously correct both amplitude and phase distortions, desired characteristics for pattern recognition applications.

Automatic target tracking is the topic of the next paper. In this paper, Zhang and Karim describe a real-time system for tracking objects in a video stream obtained from a moving airborne platform. The proposed tracking algorithm is based on the general framework of spatiotemporal segmentation followed by temporal tracking, and is capable of tracking both moving and stationary objects while accommodating objects of various types and sizes including point objects.

Temporal pattern recognition is the topic on the next paper. Zou et al. propose an ultrafast temporal pattern generation and recognition using femtosecond laser technology. By optimizing the phase of the ultrafast temporal pulse in space, they realize the controllable temporal shape in the time domain, perform ultrafast temporal pattern recognition with frequency-resolved optical gating (FROG) technique, and obtain the FROG trace for one pulse.

The next paper by Kim and Sohn deals with disparity estimation using a region dividing technique and energybased regularization. A two-stage algorithm is proposed for locating smooth and detailed disparity vector fields in a stereo image pair. Experimental results show how the proposed algorithm provides accurate and spatially correlated disparity vector fields in various types of stereo images, even in the case of images with large displacements.

The subsequent paper by Chang and Chen presents a 
new texture-based feature extraction coding method, called gradient texture unit coding (GTUC), for capturing gradient changes in a texture unit. By normalizing a texture spectrum as a probability distribution, this paper further develops an information divergence based discrimination criterion to measure the discrepancy between two texture spectra.

In the last paper of this special section, Bucholcer investigates the nonlinear transformation of a complex function for pattern recognition applications. This paper provides precise analysis of changes of complex functions nonlinearly transformed by a complex transfer characteristic. The author believes this analysis is more complete than any other analysis available on this particular topic, and it may help interpret correlation-based pattern techniques more accurately, leading to new applications.

This special section is well balanced and reports on the many ongoing efforts in pattern recognition techniques, devices, and algorithms at different academic, industrial, government, and defense research labs.

We would like to thank the authors for the contribution of their papers, the reviewers for their dedication and timely review, and the Optical Engineering staff for ensuring timely production of this special section.

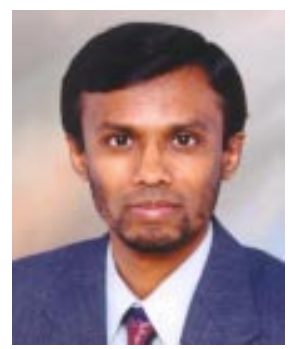

Mohammad S. Alam received his BS and MS degrees in electrical engineering from Bangladesh University of Engineering and Technology in 1983 and 1985, his MS degree in computer engineering from the Wayne State University in 1989, and his $\mathrm{PhD}$ degree in electrical engineering from the University of Dayton in 1992. Dr. Alam was on the faculty at Purdue University, Fort Wayne, Indiana from 1992 to 1999 and at the University of Alabama from 1999 to 2001. He served as a graduate faculty member of both Purdue University and Indiana University during his tenure at Purdue University, Fort Wayne. Dr. Alam joined the University of South Alabama in May 2001 as chair and professor of electrical and computer engineering. His research interests include ultrafast computer architectures and algorithms, digital/optical signal and image processing, pattern recognition, fiber-optic communication systems, infrared systems, microprocessor interfacing, and digital system design. He is the author or co-author of more than 250 published papers, including 100 articles in refereed journals and
10 book chapters. He served as the editor of the reference book of Selected Papers on Real Time Optical Pattern Recognition Using Joint Transform Correlation (SPIE Press, 1999). He serves as an associate editor for the International Journal of Science and Technology, and has served as a guest editor for Optical Engineering and the Journal of Applied Optics. Dr. Alam served as the principal or co-principal investigator of research projects totaling nearly 12 million dollars. His research work has been supported by federal agencies such as the National Science Foundation, Federal Aviation Administration, Department of Energy, Army Research Office, Air Force Office of Scientific Research, and Wright Patterson Air Force Base as well as by industry such as ITT industry and Radiance Technology, Inc. He is the recipient of numerous excellence in research, teaching and service awards including the Phi Kappa Phi 2003 Researcher of the Year Award and 1998 Outstanding Engineer Award from Region IV of IEEE. Dr. Alam has presented over 50 invited papers, seminars, and tutorials at international conferences and research institutions in the USA and abroad. He is a fellow of the Optical Society of America, a fellow of SPIE-The International Society for Optical Engineering, a senior member of the Institute of Electrical and Electronics Engineers, a member of the American Society for Engineering Education, and a member of the American Institute of Physics.

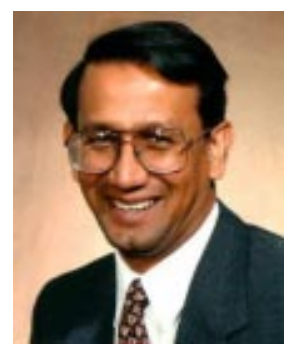

Mohammad A. Karim is the vice president for research at Old Dominion University. He received his BS in physics in 1976 from the University of Dhaka, Bangladesh, and MS degrees in both physics and electrical engineering and $\mathrm{a} P \mathrm{Ph}$ in electrical engineering from the University of Alabama respectively in 1978, 1979, and 1981. Prior to becoming a dean, he served as department head of electrical and computer engineering at the University of Tennessee in Knoxville, and as chairperson of electrical and computer engineering as well as founding director of the electrooptics program at the University of Dayton in Ohio. Dr. Karim is the author of the books Digital Design: A Pragmatic Approach, ElectroOptical Devices and Systems, Optical Computing: An Introduction, Electro-Optical Displays, and Continuous Signals and Systems Using MATLAB, six book chapters, and over 320 papers. He is the North American editor of Optics \& Laser Technology, an associate editor of the IEEE Transactions on Education and serves on the editorial board of Microwave and Optical Technology Letters. He has served as guest editor for thirteen journal special issues. Dr. Karim is a fellow of the Optical Society of America, SPIE-The International Society for Optical Engineering, and the Bangladesh Academy of Sciences, a distinguished fellow of the University of Alabama College of Engineering, a senior member of IEEE, and a member of the American Society of Engineering Education. 